\title{
THE NUMERICAL SIMULATION TECHNIQUES RESEARCH AND PRELIMINARY EXPERIMENTAL VALIDATION OF THE START CHARACTERISTICS FOR A TWO-DIMENSIONAL HYPERSONIC INLET
}

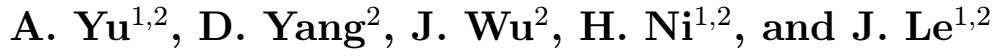 \\ ${ }^{1}$ Science and Technology on Scramjet Laboratory \\ CARDC, 621000, China \\ ${ }^{2}$ Airbreathing Hypersonic Technology Research Center \\ CARDC, 621000, China
}

\begin{abstract}
The paper presents the research of the numerical simulation techniques and the preliminary experimental validation on the start characteristics for a typical two-dimensional (2D) hypersonic inlet. In order to obtain the start and unstart hysteresis loop, numerical simulations methods using the incoming flow field, the zero-velocity flow field, and the latest convergent flow field as the initialized flow field separately are adopted to calculate the inlet start characteristics until the flow fields converge. The calculation software is AHL3D, a parallel computational fluid software self-developed by CARDC. The numerical methods also include the method from unstart flow field to start one with Mach-numbers gradually increasing till the inlet is start and the method from start flow field to unstart one with Mach-numbers decreasing till the inlet is unstart. According to the comparison with different initialized flow field and with different step-size of Mach-number, the inlet start characteristics are obtained and the numerical techniques are analyzed. Based on the comparison and the analysis, it is suggested that the numerical techniques of inlet start characteristics should use the zero-initialized flow field with one step to the final Mach-numbers to predict the minimal self-start Mach-number of the inlet and use the incoming flow-initialized flow field to predict the minimum start Mach-number. The results show that the numerical techniques are high-efficient and easily operational. To verify the effect of the numerical techniques, wind tunnel tests are arranged to research the start characteristics of a typical 2D hypersonic inlet. The preliminary experimental result shows that the numerical techniques of predicting inlet start characteristics are consistent with the experiments very well, which means that the application of the numerical techniques can be further carried out for such hypersonic inlets as mentioned in the current paper.
\end{abstract}

(C) The Authors, published by EDP Sciences. This is an open access article distributed under the terms of the Creative Commons Attribution License 4.0 (http://creativecommons.org/licenses/by/4.0/). 


\section{NOMENCLATURE}

\begin{tabular}{|c|c|}
\hline $\mathrm{Ma}$ & Mach-number \\
\hline $\mathrm{Ma}_{0}$ & Mach-number of the incoming flow \\
\hline $\mathrm{Ma}_{L}$ & Minimal Ma for an inlet to keep starting \\
\hline $\mathrm{Ia}_{R}$ & Minimal self-start Ma of an inlet \\
\hline ccelerate-start & $\begin{array}{l}\mathrm{Ma}_{0} \text { gradually increases till the inlet flow field turns to } \\
\text { start state }\end{array}$ \\
\hline Onverged-init & $\begin{array}{l}\text { A process to initial the flow field, or an initial flow field, } \\
\text { of which the flow field has converged at a Ma }\end{array}$ \\
\hline Convergent-start & $\begin{array}{l}\text { The flow field has already converged, and the re- } \\
\text { sult state of the inlet is starting, corresponding to } \\
\text { Converged-init }\end{array}$ \\
\hline Convergent-unstart & $\begin{array}{l}\text { The flow field has already converged, and the re- } \\
\text { sult state of the inlet is unstarting, corresponding to } \\
\text { Converged-init }\end{array}$ \\
\hline Decelerate-unstart & $\begin{array}{l}\mathrm{Ma}_{0} \text { gradually decreases till the inlet flow field turns to } \\
\text { unstart state }\end{array}$ \\
\hline Direct-start Ma & $\begin{array}{l}\mathrm{Ma}_{0} \text {, with which the flow is initialized, at which the } \\
\text { convergent flow of the inlet always keeps starting, cor- } \\
\text { responding to far-init }\end{array}$ \\
\hline Direct-unstart Ma & $\begin{array}{l}\mathrm{Ma}_{0} \text {, with which the flow is initialized, at which the } \\
\text { convergent flow of the inlet turns to unstart at last, } \\
\text { corresponding to far-init }\end{array}$ \\
\hline far-init & $\begin{array}{l}\text { A process to initial the flow field, or an initial flow field, } \\
\text { of which the Mach-number is initialized to be } \mathrm{Ma}_{0}\end{array}$ \\
\hline Zero-init & $\begin{array}{l}\text { A process to initial the flow field, or an initial flow field, } \\
\text { of which the Mach-number is initialized to be zero }\end{array}$ \\
\hline Zero-start Ma & $\begin{array}{l}\mathrm{Ma}_{0} \text {, at which the convergent flow of the inlet can self- } \\
\text { start with the initial flow of zero velocity, corresponding } \\
\text { to zero-init }\end{array}$ \\
\hline Zero-unstart Ma & $\begin{array}{l}\mathrm{Ma}_{0} \text {, at which the convergent flow of the inlet is un- } \\
\text { started with the initial flow of zero velocity, correspond- } \\
\text { ing to zero-init }\end{array}$ \\
\hline
\end{tabular}

\section{INTRODUCTION}

To predict the start characteristics for a fixed-geometric hypersonic inlet with a wide working range of $\mathrm{Ma}_{0}$, especially to predict the self-start characteristics, is an important step for an air-breathing hypersonic inlet in practical applications. The main purpose of thus prediction is to obtain the start hysteresis loop and the start boundary as well. Researches $[1,2]$ show that the hysteresis loops at 
present are obtained not only by wind tunnel tests $[1,3-5]$, but also by numerical simulation [6-12] as follows.

Neaves et al. [6] present a time-accurate high-speed flow calculation using a three-dimensional (3D) adaptive mesh algorithm with a time accurate implicit Navier-Stokes solver. The calculations they accomplished are the steady- and unsteady-state ones for a 3D inlet unstart due to a combustor perturbation simulated by closing a diffuser ramp which raises exit pressure. The computational results demonstrate the ability to calculate $3 \mathrm{D}$ high-speed inlet unstarts with a solution adaptive mesh algorithm. However, the document does not focus on the start characteristics of the inlet without combustor working.

Timofeev et al. [7] provided a summary of their researches on the application of locally adaptive unstructured unsteady 2D and 3D Euler numerical codes to the starting of hypersonic inlets. They carried out REST inlet computations for a range of Mach numbers under a sudden insertion into a hypersonic flow. The method of different modes of inlet starting under different initial conditions can be a good reference for current paper.

Veillard et al. [11] demonstrated a method for obtaining the limiting contraction for supersonic intake-starting via overboard spillage for a simple ramp-type intake family. The document predicted starting characteristics compared favorably with 2D inviscid numerical simulations, or the Kantrowitz starting theory. Analytical self-starting predictions were well confirmed by numerical simulation. But the inviscous results in the paper may overvaluate the theoretical guidance to the designs of practical intake.

Barber et al. [9] used two different computational fluid dynamics (CFD) codes, Choi's UTNS and NASA's CFL3D, to model complex hypersonic inlet starting phenomena. The document simulated various free-stream Machnumbers to determine whether the codes would predict consistent starting results. In order to obtain convergent, high-speed, viscous solutions, the paper points out that inviscid or Euler calculations should not be used to assess starting performance and that the choice of turbulence model can have a significant impact on the prediction of shock-wave-boundary-layer interaction. Yet, the research needs to be investigated further before the codes can be reliably used for time accurate inlet start access.

Yuan and Liang [8] adopted quazi-steady method to calculate the unstart performance for a hypersonic side-wall inlet model. The paper uses NAPA CFD software to calculate the inlet start characteristics with setting a series of Mach-numbers as incoming flow Mach-numbers (i. e., $\mathrm{Ma}_{0}$, see the Nomenclature above). The result of the document shows that the Mach-number of the inlet restart of the research may probably not be the self-started Mach-number. Yuan and Liang [10] also analyzed the characteristics of restart performance for a hypersonic inlet, adopting a method of gradually increasing $\mathrm{Ma}_{0}$. This method is believed to be a safe one to obtain restart Mach-number, or the self-start Mach-number. In fact, this method is adopted in the present paper too. 
He et al. [12] launched a lot of work and pointed out that for self-start simulation of a hypersonic inlet with variable incoming flow conditions, steady calculations give the same result as the unsteady ones do. The CFD software used in the research is AHL3D, which will be used in the present work too.

Based on the documents mentioned above, it can be inferred that the method of using steady numerical simulation to predict start characteristics for hypersonic inlets is nearly the same reasonable as that of using unsteady numerical simulation, that the initial flow of the inlet plays a prominent role in the numerical prediction of inlet start characteristics, and that the gradually changing $\mathrm{Ma}_{0}$ has a significant effect on the final result. For example, the Mach-number of incoming flow(i.e., $\mathrm{Ma}_{0}$ ) can be set to a low supersonic value and after the flow field converges, an unstart flow field can be achieved. Then, with the gradually increasing $\mathrm{Ma}_{0}$ and with the marching of unsteady calculation, the unstart inlet flow field will change little by little till the inlet starts. Thus, the minimal self-start Ma is obtained. Subsequently, the $\mathrm{Ma}_{0}$ can be decreased gradually and with steady or unsteady calculating till the inlet turn back to unstart, thus, the minimal Ma for the inlet to be able to start is obtained too. Considering that the unsteady calculation has weakness including the massive workload and the long-term calculation, which are usually very intolerant, the steady calculation will be carried out instead in the present paper.

On the summarization of the work in $[13,14]$, the present work finds that the steady calculation can also be used to predict the inlet start characteristics, particularly, for any $2 \mathrm{D}$ hypersonic inlets, through a large amount research practice. It is shown that not only $\mathrm{Ma}_{0}$ determines the calculation result of the inlet state but also the initialized flow field does the same effect. How to affect the inlet state will be discussed in the current paper. Based on the analysis and research, the present work brings about many definitions of Mach-numbers relevant to inlet start and to calculating process, as well as the numerical techniques to quickly predict inlet start characteristics. At last, some experiments are designed and launched to validate the numerical techniques of predicting inlet start characteristics.

\section{THE START HYSTERESIS LOOP AND THE RELEVANT MACH-NUMBERS}

The start hysteresis loop is a typical and common aerodynamic phenomenon for any hypersonic inlets so long as internal contraction exists, no matter how little the internal contraction is. In general, the start hysteresis loop of the inlet can be shown in the following forms: Ma hysteresis loop, angle of attack hysteresis loop, variable-geometric hysteresis loop, etc. Among them, the Ma hysteresis loop is the typical and basic one, which is shown in Fig. 1. 


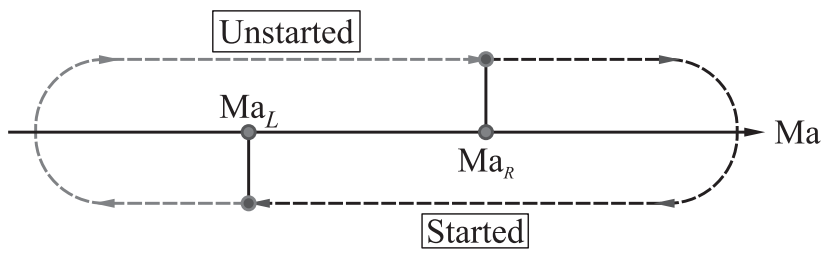

Figure 1 Start Ma hysteresis of a hypersonic inlet

In the figure, the horizontal axis is the Ma axis, and the loop with dashed line of which half is in grey and half is in black is the start Ma hysteresis loop mentioned above. Along the loop in the clockwise direction, with the increase of Ma, an inlet will quit unstart and enter a status of start. The Mach-mumber at the division point, shown as $\mathrm{Ma}_{R}$ in the figure, corresponds to the minimal self-start Ma. Meanwhile, along the same loop in the same direction, with the decrease of $\mathrm{Ma}$, a started inlet will switch to unstart. At this time, Ma at the division point, shown as $\mathrm{Ma}_{L}$ in the figure, is the minimal start Ma for an inlet to keep starting.

There are several significances about a start Ma hysteresis loop of an inlet. Firstly, it gives two boundaries. One is the minimal Ma, say, $\mathrm{Ma}_{L}$ in Fig. 1, at which the inlet can keep starting. The other is the minimal Ma, say, $\mathrm{Ma}_{R}$ in Fig. 1, at which the inlet can self-start. Obviously, $\mathrm{Ma}_{L}$ is less than $\mathrm{Ma}_{R}$; so, an inlet which can start at a lower Ma not less than $\mathrm{Ma}_{L}$, will not start until the $\mathrm{Ma}_{0}$ is increased to $\mathrm{Ma}_{R}$. This is the very reason why the inlet start hysteresis happens.

Secondly, $\mathrm{Ma}_{L}$ and $\mathrm{Ma}_{R}$ divide the whole Ma axis into three parts. In the left part with $\mathrm{Ma}<\mathrm{Ma}_{L}$, the inlet will always unstart, while in the right part with $\mathrm{Ma}>\mathrm{Ma}_{R}$, the inlet will always start. In the middle part with $\mathrm{Ma}_{L}<$ $\mathrm{Ma}<\mathrm{Ma}_{R}$, the inlet may either start or unstart.

Thirdly, the hysteresis loop is separated into two parts by $\mathrm{Ma}_{L}$ and $\mathrm{Ma}_{R}$, corresponding to unstarted state and started state, respectively. When the Ma is increased or decreased, so long as it does not cross over $\mathrm{Ma}_{L}$ or $\mathrm{Ma}_{R}$, whether the inlet is started or unstarted is decided by the nearest state just before, in fact, the state of the inlet is the same as the previous one.

Fourthly, only when $\mathrm{Ma}_{0}$ increases and crosses over $\mathrm{Ma}_{R}$, an unstarted inlet can switch to the started state. If $\mathrm{Ma}_{0}$ decreases and crosses over $\mathrm{Ma}_{L}$, a starting inlet will switch to unstart at once.

To predict the start characteristics of an inlet is just to predict the two Mach-numbers: the minimal Ma for an inlet to keep starting and the minimal Ma for an unstarting inlet to turn to start. In order to be convenient to refer in the present work, the terms $\mathrm{Ma}_{L}$ and $\mathrm{Ma}_{R}$ are used here to represent them, respectively. 


\section{THE GEOMETRIC MODEL AND THE CALCULATION METHOD}

The model of the geometry is a hypersonic inlet self-designed by CARDC, with a designing software based on parallel CFD calculation environment and multiple objectives optimization [15]. The model is a $2 \mathrm{D}$ inlet. Figure $2 a$ gives the main dimension at the symmetry.

The calculation software used in the paper is AHL3D, a parallel CFD software self-developed by CARDC independently. AHL3D solves the time-dependent, Reynolds-averaged Navier-Stokes equations for turbulent, compressible flows using a finite-volume, time-marching approach on multizone, structured grids. Spatial accuracy is formally second order using the steger-warmming flux-difference splitting upwind formulation for inviscid flux-mode. Steady flows are simulated through an iterative process using local time stepping. Unsteady flows are simulated through a second-order marching in time. Turbulence models can be chosen from Spalart-Allmaras (S-A) one-equation model, or $k-e / k-\omega$ two-equation models. AHL3D is capable of solving the flows of speeds ranging from low sub-

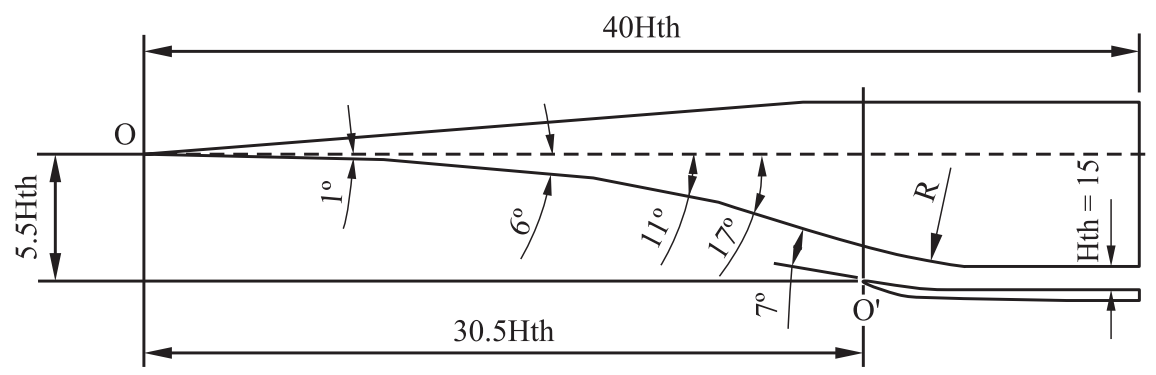

(a)

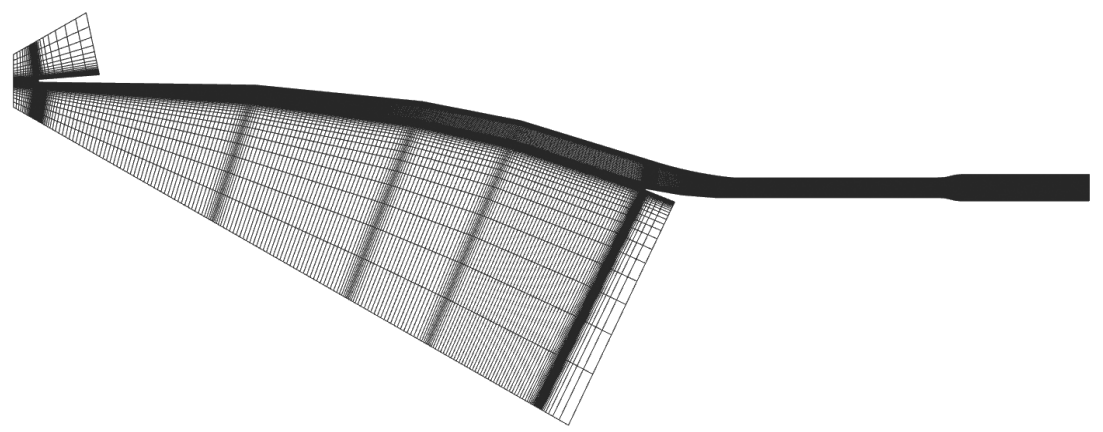

(b)

Figure 2 The main dimensions at the symmetry $(a)$ and the 2D mesh of the model $(b)$ 
sonic to hypersonic, especially, for supsonic combustion flow. At high temperature, AHL3D has models to simulate high-temperature effects in air with specific heat as a function of temperature. For the current calculation, the solver used is based on Navier-Stokes coupled solution algorithm, adopting time-dependent method to march to a steady state with a turbulent $k-\omega$ shear stress transport model and a material of ideal gas.

In order to reduce the calculation workload, the $2 \mathrm{D}$ multiblock structured mesh at the symmetry plane of the model is generated. The total number of the rectangular cells is about 54.3 thousand. Figure $2 b$ shows the structured mesh generated for the prediction of $\mathrm{Ma}_{L}$ and $\mathrm{Ma}_{R}$ in the hysteresis (a 3D mesh will be generated and used later to guide the verifying experiment). The calculation domain to be discretized is also shown in Fig. $2 b$. The boundary conditions include free incoming flow, nonslip, and adiabatic wall condition.

In the calculation, three processes are carried out in the present paper. The first process is to initialize the whole domain using the incoming flow and then march the calculation till the flow converges. The second process is to use a certain convergent flow as its initialized one and set the incoming flow condition, then march the calculation till the flow converges. The third process is to initialize the whole flow with a velocity of zero, and set the incoming flow condition, then march the calculation till the flow field converges. Whether the inlet starts or not can be judged by the convergent flow field; thus, the relationship between $\mathrm{Ma}_{0}$ at present and the state of the inlet can be built.

\section{THE SOLUTION OF Ma $\mathrm{Ma}_{L}$ AND Ma}

\subsection{The Solution of Direct-Start Ma and Direct-Unstart Ma}

The present paper chooses a specific $\mathrm{Ma}$ as the incoming flow $\mathrm{Ma}$, or $\mathrm{Ma}_{0}$, and initializes the inlet domain with it. The calculation does not stop until the flow converges. Judged from the convergent flow, if the inlet is starting, $\mathrm{Ma}_{0}$ which is used to initialize the domain is defined to be direct-start Ma. Otherwise, if the inlet is unstarting, then the $\mathrm{Ma}_{0}$ is defined to be direct-unstart Ma. It is obvious that the direct-start $\mathrm{Ma}$ corresponds to a Ma at which the inlet can keep starting and that the direct-start Ma has a minimal value while the direct-unstart Ma has a maximal value. Although the maximal direct-unstart $\mathrm{Ma}$ is less than the minimal direct-start Ma, they are almost the same as each other. It can be inferred that the minimal direct-start Ma may possibly be the same as the value of $\mathrm{Ma}_{L}$ in Fig. 1.

The direct-start Ma has a minimal value. To predict this value, many calculations with different $\mathrm{Ma}_{0}$ are needed. Here, in the current paper, $\mathrm{Ma}_{0}$ represents 


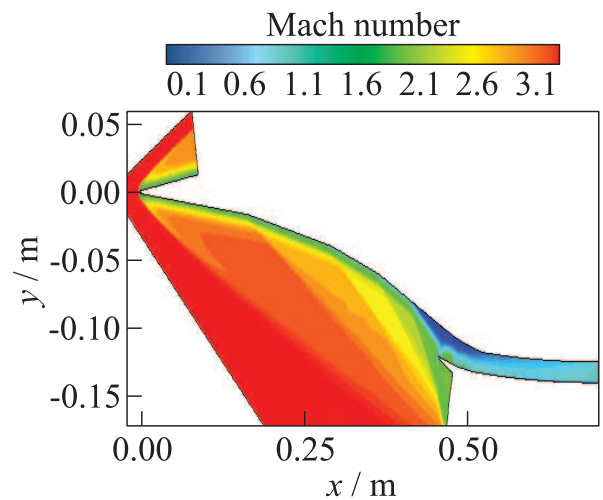

(a)

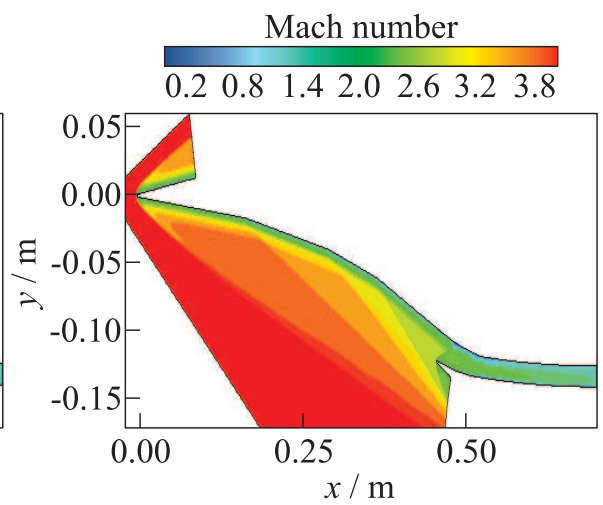

(b)

Figure 3 The example for the solution of direct-unstart Ma and direct-start Ma: (a) far-init $\mathrm{Ma}_{0}=3.5$; and $(b)$ far-init $\mathrm{Ma}_{0}=4.0$

the incoming flow Mach-number(see the Nomenclature above). Figure 3 gives an example for the solution of minimal direct-start Ma.

From the calculation, it can be seen that the minimal direct-start $\mathrm{Ma}$ is between 3.5 (Fig. $3 a$ ) and 4.0 (Fig. $3 b$ ).

\subsection{The Solution of $\mathrm{Ma}_{L}$}

$\mathrm{Ma}_{L}$ means a minimal Mach-number at which the inlet still keeps starting. In the Ma start hysteresis loop, $\mathrm{Ma}_{L}$ corresponds to the boundary Mach-number for a hypersonic inlet to switch from starting to unstarting.

From Fig. 1, $\mathrm{Ma}_{L}$ can be solved as follows. Firstly, try the solution of directstart Ma using a Mach-number, as $\mathrm{Ma}_{0}$, which should be greater than $\mathrm{Ma}_{R}$, usually through trial-and-error solution. Thus, a direct-start Ma and a convergentstarted flow field can be obtained. Then, decrease $\mathrm{Ma}_{0}$ but adopt the last convergent-start flow field as the initial flow field to calculate till it converges. Here, the initialized flow field is defined as Converged-Init. Continue decreasing $\mathrm{Ma}_{0}$ and calculating till the flow field converges. Thus, decelerating $\mathrm{Ma}_{0}$ will result to an unstart state of the inlet, i. e., decelerate-unstart happens. Hence, the relationship of a series of Mach-numbers and starting or unstarting inlet states is achieved. From analyzing the relationship $\mathrm{Ma}_{L}$ can be acquired. In practice, $\mathrm{Ma}_{L}$ always lies within a range. Figure 4 gives an example process for the solution of $\mathrm{Ma}_{L}$.

From the calculation process and the result shown in Fig. 4, it can be seen that $\mathrm{Ma}_{L}$ is between 3.5 and 4.0. It can also be inferred that the range of $\mathrm{Ma}_{L}$ is the same as that of direct-start Ma. 


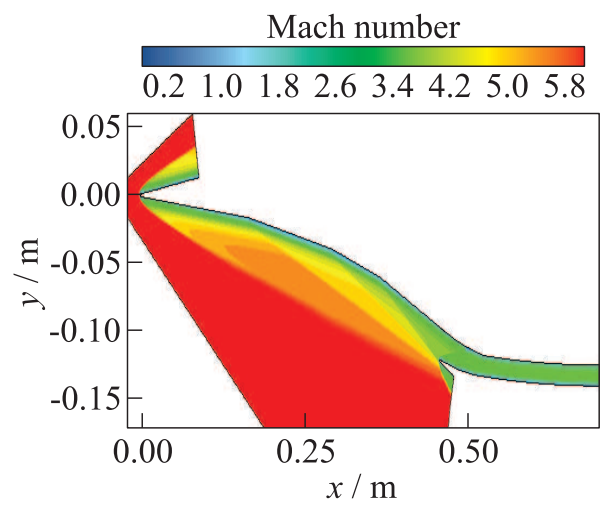

(a)

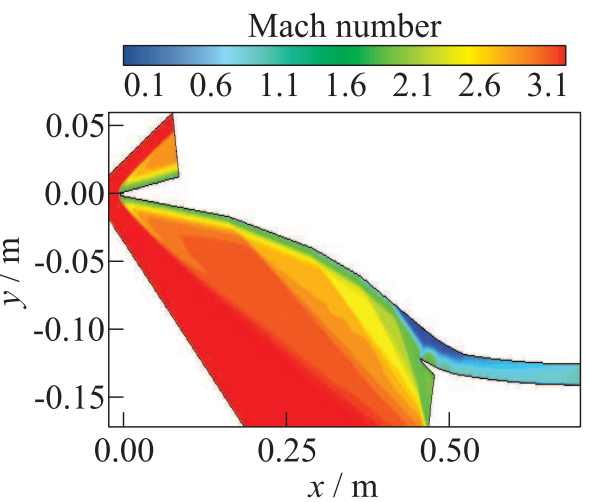

(b)

Figure 4 The example process for the solution of $\mathrm{Ma}_{L}:(a)$ direct-start $\mathrm{Ma}=6.0$; and $(b)$ from the result of $(a)$, gradually decreasing $\mathrm{Ma}_{0}$ with Ma step-size of 0.5 , till the flow filed is unstart when $\mathrm{Ma}_{0}=3.5$

\subsection{The Solution of $\mathrm{Ma}_{R}$}

As is pointed out above, $\mathrm{Ma}_{R}$ is a boundary Mach-number over which the increasing $\mathrm{Ma}_{0}$ crosses, the inlet will switch from unstarting to starting state. It is also difficult to obtain the exact value of $\mathrm{Ma}_{R}$. Instead, the range of $\mathrm{Ma}_{R}$ is obtained in practice.

From the analysis of the start Ma hysteresis loop shown in Fig. 1, the solution of $\mathrm{Ma}_{R}$ can be explained as following process. First, a direct-unstart Ma and the corresponding convergent-unstart flow field can be obtain. Second, use the convergent-unstart flow field obtained just now as the initial flow field but gradually increase the $\mathrm{Ma}_{0}$. Calculate the flow field till the solution converges. The research shows that there are two ways to set the initial flow field. One is single-step method with variable-step sizes and the other is multiple-steps method with a fixed-step size. The way of single-step method with variable-step sizes means that taking a convergent-unstarting flow field as the same initial one and parallel changing the step size to set a series of $\mathrm{Ma}_{0}$ and calculating them till the range of $\mathrm{Ma}_{R}$ is attained. The way of multiple-step method with a fixed-step size means that for every flow calculation, the initial flow field changes to the latest convergent-unstarting flow field with $\mathrm{Ma}_{0}$ increasing gradually and that all the calculations are the serial ones till the last flow field converges to a starting state. Whichever the way is, the process for the solution of $\mathrm{Ma}_{R}$ is a process using trial-error-solution.

Figure 5 gives an example of single-step method with variabl-step sizes for the solution of $\mathrm{Ma}_{R}$. From the result of Fig. 5, it can be seen that $\mathrm{Ma}_{R}$ is in the range between 5.0 and 5.1 . 


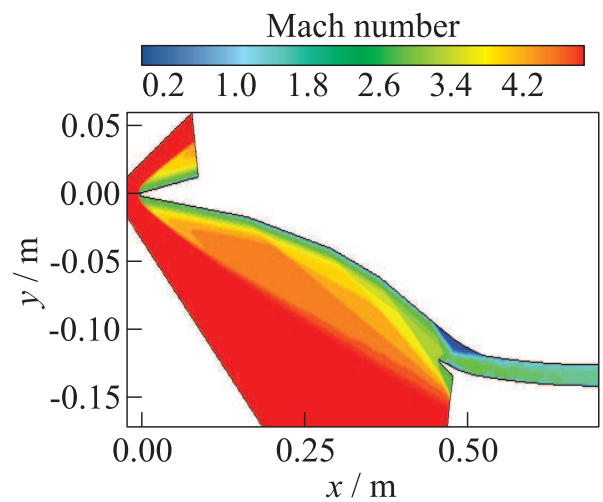

(a)

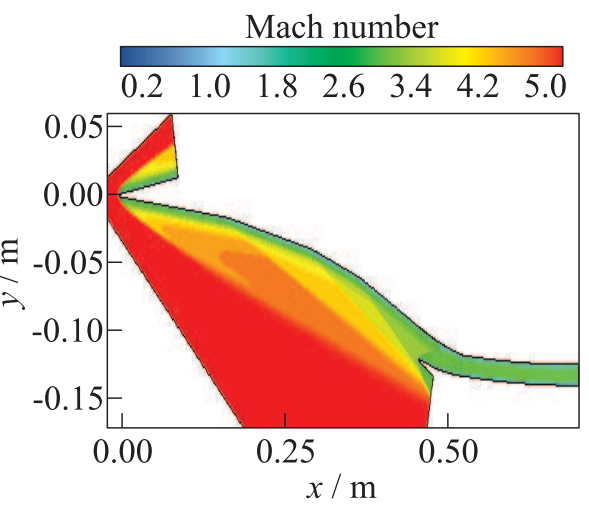

(b)

Figure 5 The example of single-step method with variable-step sizes for the solution of $\mathrm{Ma}_{R}$; converged-init $\mathrm{Ma}=3.5:(a) \mathrm{Ma}_{0}=5.0$; and $(b) \mathrm{Ma}_{0}=5.1$

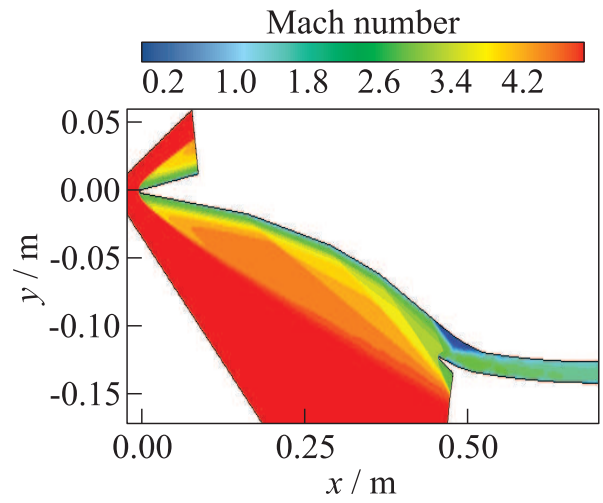

(a)

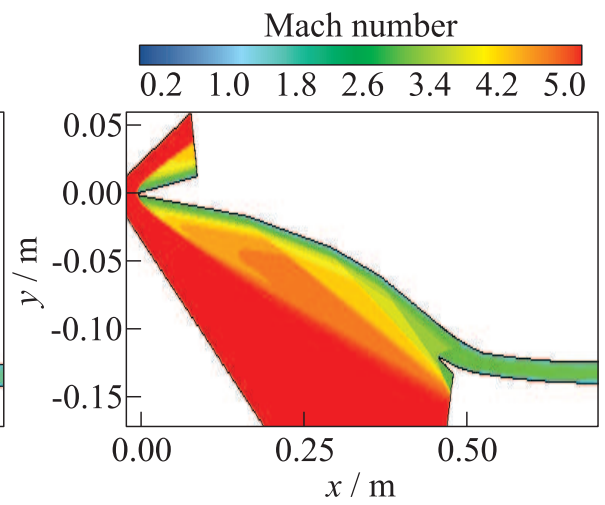

(b)

Figure 6 The example of muliple-step method with a fixed-step size for the solution of $\mathrm{Ma}_{R}$ (step size $=0.1$ ); the latest converged-init flow field, gradualy accelerating till $\mathrm{Ma}_{0}=5.0(a)$ and $5.1(b)$

Figure 6 gives an example of muliple-step with a fixed-step size for the solution of $\mathrm{Ma}_{R}$.

From the result shown in Fig. 6, it can be seen that $\mathrm{Ma}_{R}$ using muliple-step with a fixed-step size way is also in a Mach-number range between 5.0 and 5.1.

Comparing the results of these two ways, it is shown that $\mathrm{Ma}_{R}$ from the solution of multiple-step method with a fixed-step size way is almost the same as that from the solution of single-step method with variable-step sizes way. 


\subsection{The Solution of Zero-Start and Zero-Unstart Mach-Numbers}

The zero-start Ma or zero-unstart Ma solution derives from the practice.

The term zero-start Ma is defined as follows. First, initialize the flow field with a velocity of zero and then, set the $\mathrm{Ma}_{0}$ as a specific one, followed by using the steady solver to calculate the inlet flow field.

On the one hand, if the flow becomes starting, a lower Mach-number is set to be $\mathrm{Ma}_{0}$ and the calculation continues. The process goes on till the flow converges to an unstarting state.

On the other hand, if the flow becomes unstarting, a higher Mach-number is set to be $\mathrm{Ma}_{0}$ and the calculation continues too. With the process going on, a starting state flow field will be obtained sooner or later. When the flow field of the inlet is starting in these processes, $\mathrm{Ma}_{0}$ is called zero-start Ma. Otherwise, when the flow field of the inlet is unstarting, $\mathrm{Ma}_{0}$ is called zero-unstart Ma. The following calculation shows that the zero-start Ma solution can replace that of single-step or multiple-step method.

Both the zero-start Ma and the zero-unstart Ma exist in ranges. The minimal zero-start Ma is almost the same as the maximal zero-unstart Ma (in fact, former is a little greater than the latter). Figure 7 gives an example process for the solution of zero-start Ma.

The calculation shows that the zero-start Ma is between 5.0 and 5.1 and that the range of the zero-start $\mathrm{Ma}$ is the same as that of step method. This means that the minimal zero-start $\mathrm{Ma}$ is identical to the minimal value of self-start Mach-number, or $\mathrm{Ma}_{R}$ in the present paper.

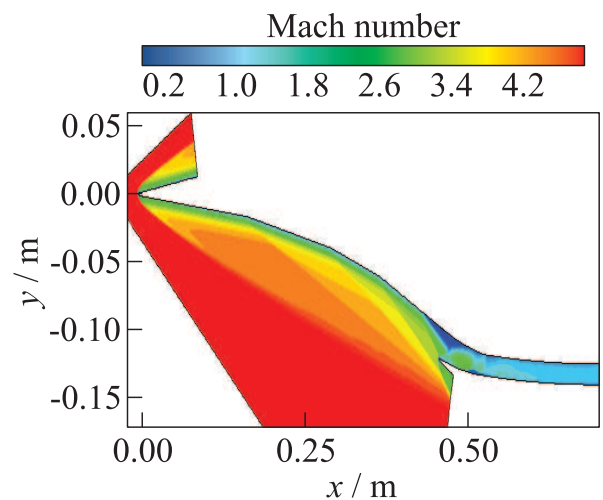

(a)

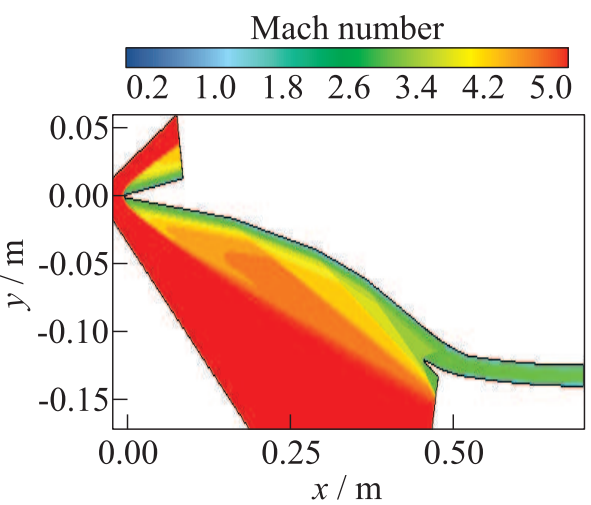

(b)

Figure 7 The example process for the solution of zero-start Ma $=5.0(a)$ and $5.1(b)$ 


\section{THE FURTHER DISCUSSION OF MACH-NUMBERS DEFINED ABOVE}

Through the results researched above, the minimal starting Ma of these solutions and processes can be listed here with each range in Table 1.

From Table 1, it can be shown that $\mathrm{Ma}_{L}$ is identical to the minimal value of direct-start $\mathrm{Ma}$ and that $\mathrm{Ma}_{R}$ is identical to the minimal value of zerostart Ma. Since the process of direct-start Ma solution is simpler than that of $\mathrm{Ma}_{L}$ solution, and the process of zero-start Ma solution is simpler than that of $\mathrm{Ma}_{R}$ solution and both the process for the solution of direct-start $\mathrm{Ma}$ and for the solution of zero-start Ma can be parallel calculated at the same time, the present research suggests that the $\mathrm{Ma}_{L}$ and $\mathrm{Ma}_{R}$ in the inlet start Ma hysteresis loop should be obtained by the solution of direct-start Ma and zerostart Ma.

The following content will further analyze the feasibility and the reliability of replacing $\mathrm{Ma}_{L}$ and $\mathrm{Ma}_{R}$ with the direct-start $\mathrm{Ma}$ and zero-start Ma, respectively, through the comparison of the pressure distribution on the body wall of the converged flow fields with different solutions.

Figure 8 gives the pressure distributions along the body wall of the convergent flow fields of the different solutions. From the figure, it can be seen that all the pressure distributions compared are totally identical with each other. Hence, $(i)$ when $\mathrm{Ma}_{0}$ is less than the minimal direct-start $\mathrm{Ma}$ or $\mathrm{Ma}_{L}$, the in-

Table 1 The range of starting Mach-number

\begin{tabular}{|c|c|c|c|}
\hline No. & $\begin{array}{c}\text { The term } \\
\text { of the minimal Ma }\end{array}$ & $\begin{array}{c}\text { The result } \\
\text { of calculation }\end{array}$ & Remark \\
\hline 1 & Direct-start Ma & $3.5 \sim 4.0$ & $\begin{array}{l}\text { Initialize the whole flow field using } \\
\text { the } \mathrm{Ma}_{0} \text { (the incoming flow Mach- } \\
\text { number) }\end{array}$ \\
\hline 2 & $\mathrm{Ma}_{L}$ & $3.5 \sim 4.0$ & $\begin{array}{l}\text { Use the started and convergent flow } \\
\text { field as the initial flow field with } \\
\text { gradually decreasing } \mathrm{Ma}_{0}\end{array}$ \\
\hline \multirow{2}{*}{3} & \multirow{2}{*}{$\mathrm{Ma}_{R}$} & $\begin{array}{l}\text { Single-step method: } \\
\quad 5.0 \sim 5.1\end{array}$ & $\begin{array}{l}\text { Use the same flow field with un- } \\
\text { starting and converging state as } \\
\text { the initial flow field, gradually de- } \\
\text { creased } \mathrm{Ma}_{0}\end{array}$ \\
\hline & & $\begin{array}{l}\text { Multiple-step method: } \\
5.0 \sim 5.1\end{array}$ & $\begin{array}{l}\text { Use the latest flow field with un- } \\
\text { starting and converging states as } \\
\text { the initial flow field, gradually in- } \\
\text { creased } \mathrm{Ma}_{0}\end{array}$ \\
\hline 4 & Zero-start Ma & $5.0 \sim 5.1$ & $\begin{array}{l}\text { Initialize the flow field velocity to } \\
\text { zero, set a series of } \mathrm{Ma}_{0}\end{array}$ \\
\hline
\end{tabular}




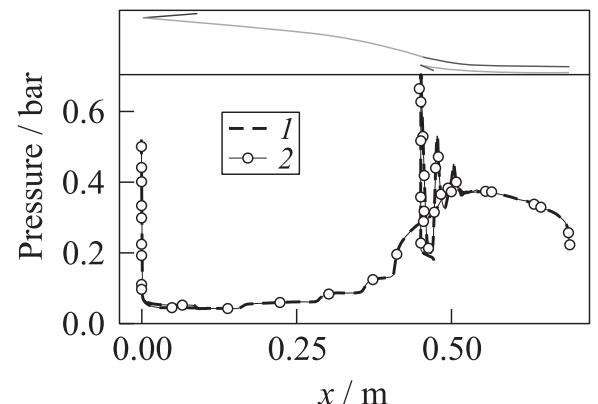

(a)

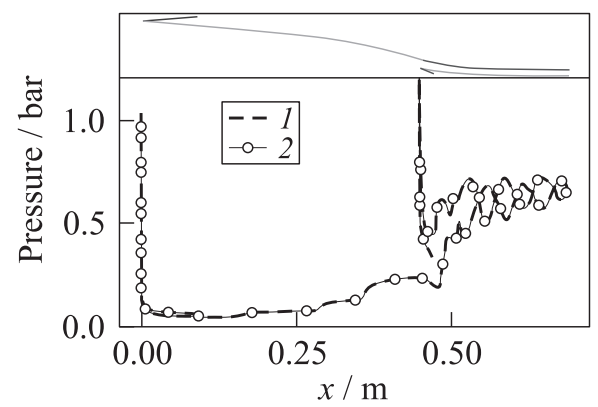

(c)

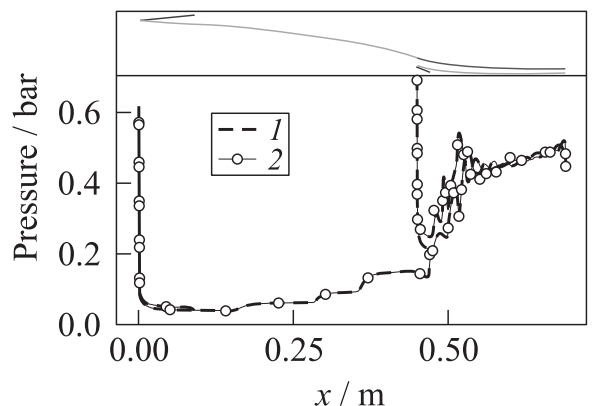

(b)

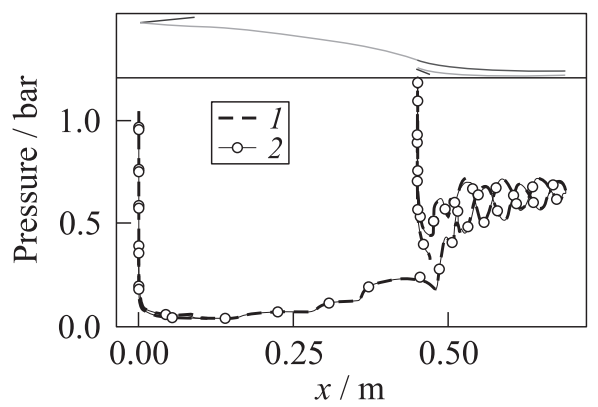

(d)

Figure 8 The comparison of pressure distribution with different solution and processes: (a) direct-start $\mathrm{Ma}=3.5$ and step-decreasing $\mathrm{Ma}_{0}$ to $3.5 ;$ (b) direct-start $\mathrm{Ma}=4.0$ and step-decreasing $\mathrm{Ma}_{0}$ to $4.0 ;(c)$ step-increasing $\mathrm{Ma}_{0}$ to 5.5 and directstart $\mathrm{Ma}=5.5 ;$ and $(d)$ zero-start $\mathrm{Ma}_{0}=5.5$ and direct-start $\mathrm{Ma}=5.5$

let flow field will not start and the pressure distribution is the same as each other at the same $\mathrm{Ma}_{0}<\mathrm{Ma}_{L}$, no matter whether the process used is the solution of direct-start Ma or the solution of $\mathrm{Ma}_{L} ;(i i)$ when $\mathrm{Ma}_{0}>\mathrm{Ma}_{L}$, the inlet flow field will always start both by the solution of direct-start Ma and by the solution of $\mathrm{Ma}_{L}$, and the two pressure distributions are the same as each other at the same $\mathrm{Ma}_{0}>\mathrm{Ma}_{L}$; (iii) when $\mathrm{Ma}_{L}<\mathrm{Ma}_{0}<\mathrm{Ma}_{R}$, the pressure distribution using step-method by solution of $\mathrm{Ma}_{R}$ is the same as that by the solution of zero-start Ma, which means the solution of zero-start Ma can converge to a correct unstarting result; and $(i \nu)$ when $\mathrm{Ma}_{0}>\mathrm{Ma}_{R}$, the inlet flow field will always start for all the solutions, and the distributions for the solution of zero-start Ma are identical to those for the solution of direct-start Ma, which reveals that the solution of zero-start Ma can converge to a correct starting result.

The double-solution phenomena with $\mathrm{Ma}_{L}<\mathrm{Ma}_{0}<\mathrm{Ma}_{R}$ have been verified by the current research too. Figure 9 gives two flow fields at $\mathrm{Ma}_{0}$ of 4.0. Figure $9 a$ 


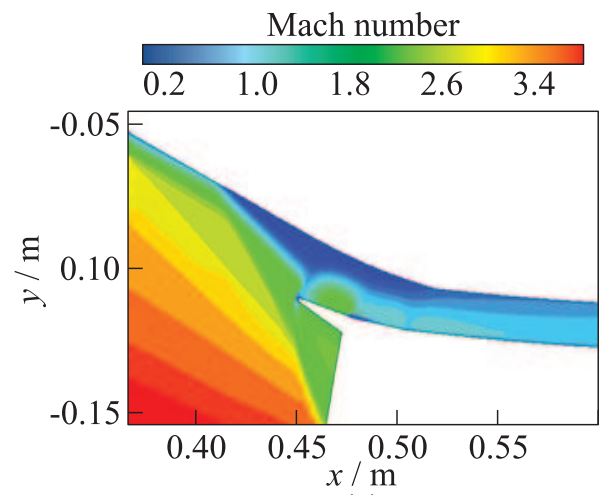

(a)

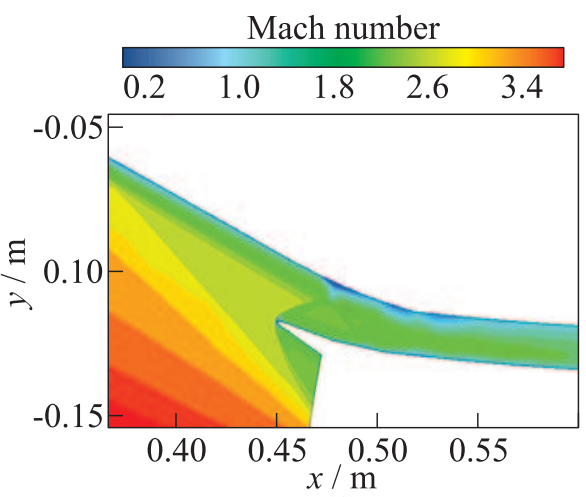

(b)

Figure 9 Two configurations in the double-solution area with the same geometry and incoming flow condition

shows an unstart inlet at $\mathrm{Ma}_{0}$ of 4.0 by the solution of zero-unstart Ma, while Fig. $9 b$ shows a start inlet at the same $\mathrm{Ma}_{0}$ as 4.0 by the solution of directstart Ma. This can further explain why there are two solutions at a Mach-number between $\mathrm{Ma}_{L}$ and $\mathrm{Ma}_{R}$ in Fig. 1.

\section{VALIDATION OF THE INLET START CHARACTERISTICS}

In order to validate the calculation techniques mentioned in this paper, some experiments are conducted to obtain the start characteristics.

\subsection{Test Model and Numerical Simulation}

The model used in the wind tunnel has the same dimension at symmetry plane as that in Fig. 2. The model has a width in the internal flow path as 8 times height of the throat and a width in the external flow path as 13.3 times height as the throat. Figure $10 a$ gives a photograph of the model in the test segment of the wind tunnel.

In order to provide the data for validation, the start characteristics are calculated using the numerical simulation techniques by solution of direct-start $\mathrm{Ma}$ and of zero-start Ma. The mesh generated here is 3D because the test model has $3 \mathrm{D}$ effects in the wind tunnel. The total amount of the multiblock structured mesh cells is about $5.4 \cdot 10^{6}$ shown in Fig. $10 b$. 


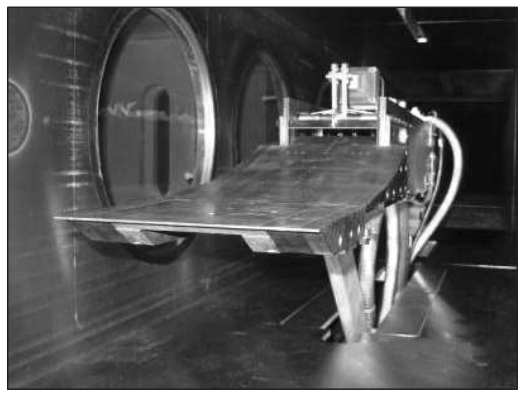

(a)

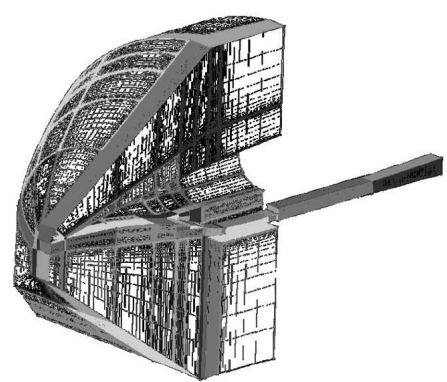

(b)

Figure 10 The photograph of the geometric 3D model in the wind tunnel $(a)$; and the $3 \mathrm{D}$ multiblock structured mesh of the inlet $(b)$

\subsection{The Wind Tunnel Experimental Results}

The experiments are conducted in $0.6 \times 0.6 \mathrm{~m}$ blowdown sub-tran-sup-sonic wind tunnel in CARDC (China Aerodynamics Research and Development Center). The nominal Mach-number is 4.0, the nominal total pressure is $6.25 \mathrm{~atm}$, and the nominal total temperature is $288 \mathrm{~K}$. Because both the start and unstart states are needed, a rotatable cowl [16] is adopted which can rotate at real-time in the experimental model.

The start and unstart states have been achieved successfully in the experiments. The typical pressure data along the flow direction are shown in Fig. 11. The results of CFD are shown in the figure too. Figure $11 a$ gives the CFD pressure distribution by the solution of direct-start Ma, and Fig. $11 b$ gives the

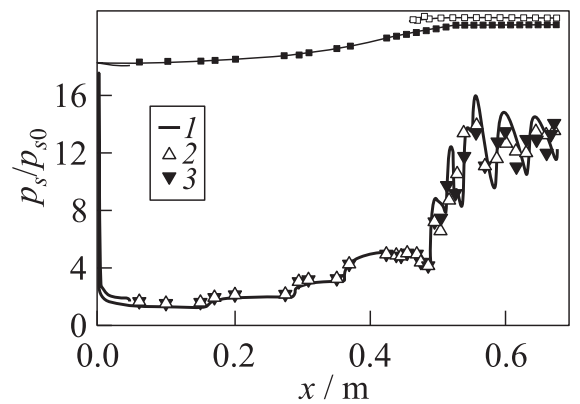

(a)

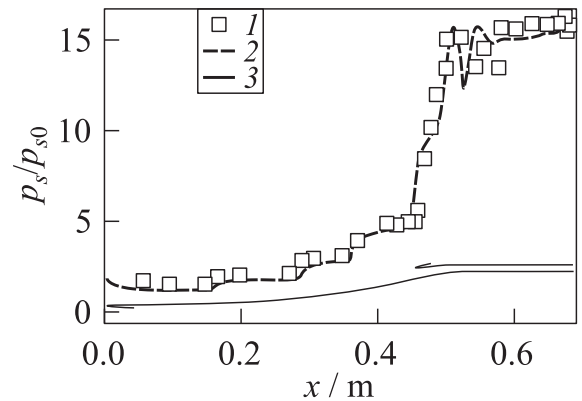

(b)

Figure 11 The comparison of pressure distribution between the calculation and experiment: $(a)$ the inlet is starting $\left(1-\right.$ wall: centerbody; $2-1$ st $=7^{\circ}$; and $3-$ $\left.2 \mathrm{nd}=7^{\circ}\right)$; and $(b)$ the inlet is unstarting $\left(1\right.$ - test $=7^{\circ} ; 2-\mathrm{CFD}$; and 3 - geometry $)$ 


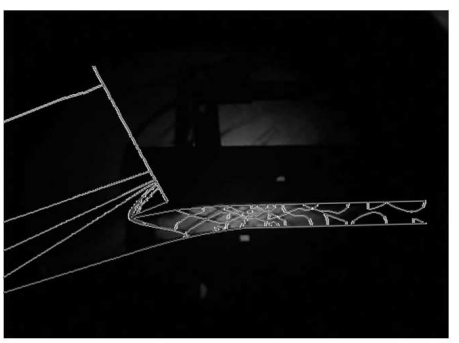

(a)

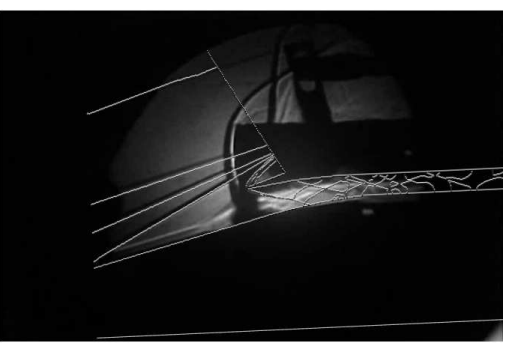

(b)

Figure 12 The wave structure comparison with calculation and experiments: $(a)$ the inlet is starting; and $(b)$ the inlet is unstarting

CFD pressure distribution by the solution of zero-unstart Ma. Comparing the pressure distribution from the experiments with that from calculation, it can be seen that the distributions are almost consistent with each other, which means that the numerical simulation techniques of direct-start Ma and zero-start $\mathrm{Ma}$ for the evaluation of inlet start characteristics are of feasibility and credibility.

Figure 12 gives the schlieren photographs of the shock structure. The figures also give the numerical schieren. Comparing these two shock wave structures in Figs. $12 a$ and $12 b$, it can be seen that the numerical simulation techniques by solution of direct-start Ma and zero-start Ma are surely precise and exact enough to predict the inlet start characteristics. This also means that the techniques of direct-start Ma and zero-start Ma presented in the paper can be used to predict the inlet start characteristics and can be developed for the future research and application.

These experiments and their data are preliminary, but the result is exciting. Further research will be carried out at the critical point between start and unstart states and on the $3 \mathrm{D}$ effect.

\section{CONCLUDING REMARKS}

Based on the analysis of the start Mach-number hysteresis loop and researches of numerical simulation processes, the present paper brings forward several concepts and solutions of some starting Mach-numbers. The conclusion drawn from the current research is as follows:

(1) the minimal Mach-number for a hypersonic inlet to keep starting and the minimal Mach-number for the inlet to self-start, corresponding to $\mathrm{Ma}_{L}$ and $\mathrm{Ma}_{R}$ in the start Ma hysteresis loop, respectively, can be calculated by using AHL3D, a CFD software self-developed by CARDC independently; 
(2) $\mathrm{Ma}_{L}$ and $\mathrm{Ma}_{R}$ in the start Ma hysteresis loop can be obtained by the solution of direct-start Ma and by the solution of zero-start Ma, respectively; and

(3) the experimental results preliminarily show that the solution of $\mathrm{Ma}_{L}$ and $\mathrm{Ma}_{R}$ in the current paper can provide a quick and convenient method to predict starting characteristics for hypersonic inlets, especially, for the $2 \mathrm{D}$ ones.

\section{REFERENCES}

1. Emami, S., C. A. Trexler, A.H. Auslender, and J.P. Weidner. 1995. Experimental investigation of inlet-combustor isolators for a dual-mode scramjet at a Mach Number of 4 NASA Technical Pater 3502.

2. Falempin, F., E. Wendling, M. Goldfield, et al. 2007. Comparative tests of adjusted inlet in blow-down and hot-shot wind tunnels. 8th Symposium (International) on Experimental and Computational Aerothermodynamics of Internal Flows Proceedings. Lyon. ISAIF8-0049.

3. Smart, M. K. 2001. Experimental testing of a hypersonic inlet with rectangular-toelliptical shape transition. J. Propul. Power 17(2):276-283.

4. Smart, M. K., and C. A. Trexler. 2004. Mach 4 performance of hypersonic inlet with rectangular-to-elliptical shape transition. J. Propul. Power 20(9.2):289-293.

5. Nan, X., K. Zhang, Z. Jin, and Y. Li. 2012. Start characteristics of hypersonic inlets with rectangular to circular shape transition at Ma 5 with attack angle $8^{\circ}$. J. Nanjing University of Aeronautics \& Astronautics 44(2):146-151. (In Chinese.)

6. Neaves, M. D., D.S. McRae, and J. R. Edwards. 2001. High-speed inlet unstart calculations using an implicit solution adaptive mesh algorithm. AIAA Paper No. 2001-0825.

7. Timofeev, E. V., P. A. Voinovich, K. Takayama, R. B. Tahir, and S. Molder. 2001. Adaptive unstructured Euler simulations of hypersonic inlet flow starting. AIAA Paper No. 2001-1896.

8. Yuan, H., and D. Liang. 2004. Characteristic analysis of unstart performance for hypersonic side-wall inlet model. J. Nanjing University of Aeronautics \& Astronautics 36(6):683-687. (In Chinese.)

9. Barber, T. J., D. Hiett, and S. Fastenberg. 2006. CFD modeling of the hypersonic inlet starting problem. AIAA Paper No. 2006-123.

10. Yuan, H., and D. Liang. 2006. Analysis of characteristics of restart performance for a hypersonic inlet. J. Propul. Technol. 127(15):390-398. (In Chinese.)

11. Veillard, X., R. Tahir, E. Timofeev, and S. Mölder. 2008. Limiting contractions for starting simple ramp-type scramjet intakes with overboard spillage. J. Propul. Power 24(5):1042-1049. 
12. He, Y., J. Wu, and A. Yu. 2014. Self-start simulation of a hypersonic inlet with variable incoming flow conditions[C]. IHFC2014-PaperList64.

13. Yu, A., and J. Le. 2005. The preliminary 2D numerical simulation research on start problem of a hypersonic inlet. 1st Chinese Conference of Hypersonic Inlets Techniques. Nanjing City, Jiangsu Province. 34-41. (In Chinese.)

14. Huang, T., A. Yu, and J. Le. 2006. The research on the start characteristics of a 2D hypersonic inlet. 12th National Shock and Shock Tube Conference. Luoyang City, Henan Province. 42-46.

15. Zhang, Y., G. Wang, and Y. He. 2007. Design and optimization of 2D hypersonic inlet. J. Aerodynamics Phys. Theory Appl. 2:82-86. (In Chinese.)

16. Yu, A., J. Le, T. Huang, and H. Ni. 2008. Experimental investigation of starting characteristics for a hypersonic inlet at Mach 4. 7th Congress of SRHRC (SinoRussia High-Speed Flow Conference). Novosibirsk, Russia. 266. 